\title{
Analysis of Ventricular Wall Motion Based on an Electromechanical Biventricular Model
}

\author{
L Xia, M Huo \\ Department of Biomedical Engineering, Zhejiang University, Hangzhou, PR China
}

\begin{abstract}
A biventricular model, which coupled the electrical property and mechanical property of the heart, was constructed and the ventricular wall motion and deformation have been simulated based on this model. The biventricular mechanical model was constructed based on composite material theory and finite element method. The excitation propagation was simulated by electrical heart model, and the resulted active forces were used to calculate the ventricular wall motion. Regional deformation and Lagrangian strain tensors were calculated during systole phase. Displacements, minimum principal strains and torsion angle were used to describe the motion of two ventricles. The results are in good accordance with solutions obtained from MR tagging images that reported in the literatures. This simulation suggests that such electromechanical biventricular model can be used to assess the mechanical function of two ventricles.
\end{abstract}

\section{Introduction}

During the past decades, many researchers devoted themselves to the biomechanical study of human heart, hoping to better understand heart dynamic properties and kinetic laws. Many mechanical heart models have been developed, but mainly focus on the left ventricle (LV). Initially, the classical mathematical models of LV were analytical ones with some simplified assumption [1-3]. With the rapid development of computer technology and medical image technology, finite element method (FEM) has become one of the main tools to study cardiovascular mechanics [4-6]. The existing LV models have described many different features of ventricular mechanics. For example, Guccione described regional heterogeneity of the fiber stress and strain in the LV [5] and Bovendeerd proved that the distributions of the active muscle fiber stress and muscle fiber strain across the LV wall are sensitive to the fiber orientation [6].

As mentioned above, previous mechanical heart models were mainly constructed for analysis of the LV behavior, and the right ventricle (RV) was not included because of its complex geometry structure. The motion of the healthy RV and its alteration due to disease are not currently well understood. Consider that the RV also plays an important role in normal and abnormal hemodynamics, a biventricular model should be constructed and used to study the mechanical property of the heart. The key idea of this paper is to build a biventricular model which contracts under electrical excitation. In addition, the ventricular wall motion and deformation will be simulated based on this model.

\section{Method}

\subsection{Model construction}

It is well known that the myocardial fiber structure of the heart plays a critical role in electrical propagation and force production. Myocardial electrical propagation is anisotropic, with the spread of current greatest in the direction of the long axis of the fiber. Fiber orientation is also an important determinant of myocardial stress and strain [7]. According to the literatures [8,9], we constructed the following fiber orientation in the biventricular model: in the left and right ventricular free wall, fiber orientation typically varies from $-60^{\circ}$ at the epicardium to $+90^{\circ}$ at the endocardium, whereas in the septal wall the fiber angle ranges from approximately $90^{\circ}$ at the right ventricular endocardium to around $+80^{\circ}$ at the left ventricular endocardium. At the junction of ventricular free wall and the septum, we used a certain interpolation function to make the junction smoothly.

As Fig. 1 described, the biventricular model was constructed on the basis of LFX model [10]. Based on the image data of the human body and heart sections, the LFX model was reconstructed as a 3-D array of approximately 65000 cell units spaced $1.5 \mathrm{~mm}$ apart. It was proved that this model is efficient with reasonable accuracy [10]. After the cardiac electrical excitation series was gotten, we could compute the active forces of myocardium [11].

Then, a 3-D finite element electro-mechanical model of biventricle was constructed based on composite theory [11]. 3-D 8 nodes isoparametric element was the basic element, and there were total 13-layer nodes, 1489 brick elements, and 5937 degrees of freedom (Fig. 2). 
We used the special isoparametric method [11] to discretize the two ventricles. Each element could accommodate several layers with different ply orientations, thus the active force vectors are unidirectional in a layer. After the active forces per element were calculated, FEM could be used to analyze the displacements of nodes in the biventricular model. Finally, the three-dimensional displacement field of two ventricles could be calculated and then used to compute Lagrangian strain tensor at each material point in the myocardium as a function of time.

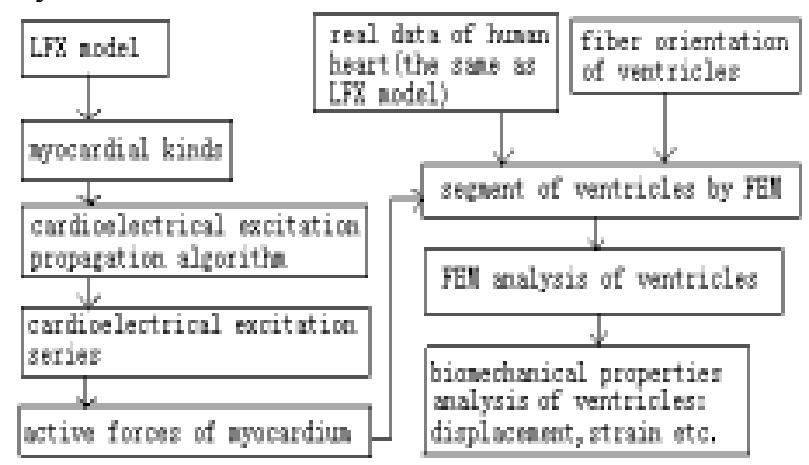

Fig. 1 The construction flow chart of biventricular model

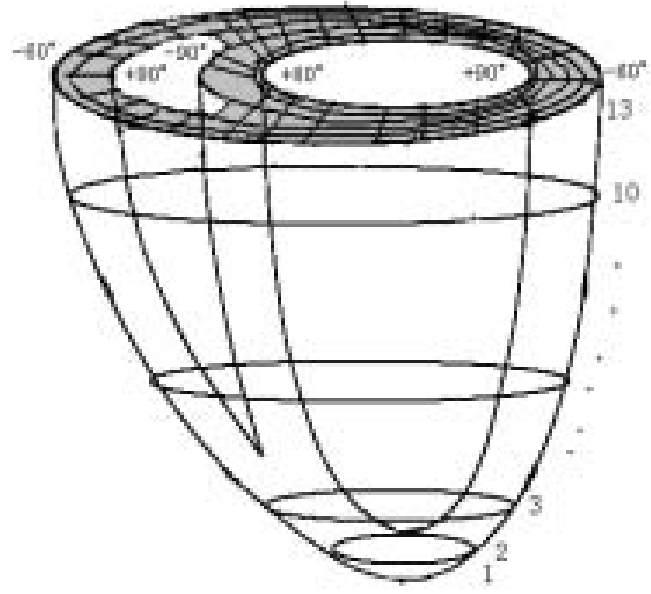

Fig. 2 The FEM segments of the biventricular model

\subsection{Deformation analysis}

Lagrangian strain: Strain analysis is a method to describe the internal deformation of a continuum body. It is an appealing tool to study and quantify myocardial deformation. The Lagrangian strain $\mathrm{E}$ is used to describe systolic deformation in a region surrounding a point in the heart wall relative to its initial position at end-diastole. $\mathrm{E}$ was calculated from the relationship $\mathrm{E}=1 / 2\left(\mathrm{~F}^{\mathrm{T}} \mathrm{F}-\mathrm{I}\right)$, where the superscript $\mathrm{T}$ represents the matrix transpose, $\mathrm{F}$ represents deformation gradient tensor, and I, the identity matrix. The deformation gradient tensor $F$ can be calculated for the relationship $F_{p q}=\partial x_{p} / \partial X_{q}$, where $\mathrm{X}$ and $\mathrm{X}$ represent the initial and final position of a point in the material respectively, the subscripts $\mathrm{p}$ and $\mathrm{q}$ range from 1 to 3 and denote one of the 3D Cartesian coordinates. The tensor, $\mathrm{F}$, includes both the rotation and deformation around a point in the material [12].

Torsion angle: This measurement describes the rotation between base and apex that develops during systole, as described by Buchalter et al. [13]. Two short axis slices are compared, the base and apical slice. To eliminate rigid body rotation, only end-systolic images are compared. Torsion is defined as the difference at endsystole in the position of a basal material point and the corresponding material point on a succeeding slice expressed as an angle of rotation. This angle represents the rotation of one point with respect to the other point. As shown in Fig. 3, increasing angle (positive sign) indicates counter clockwise rotation of the apex when viewed looking towards the base [14].

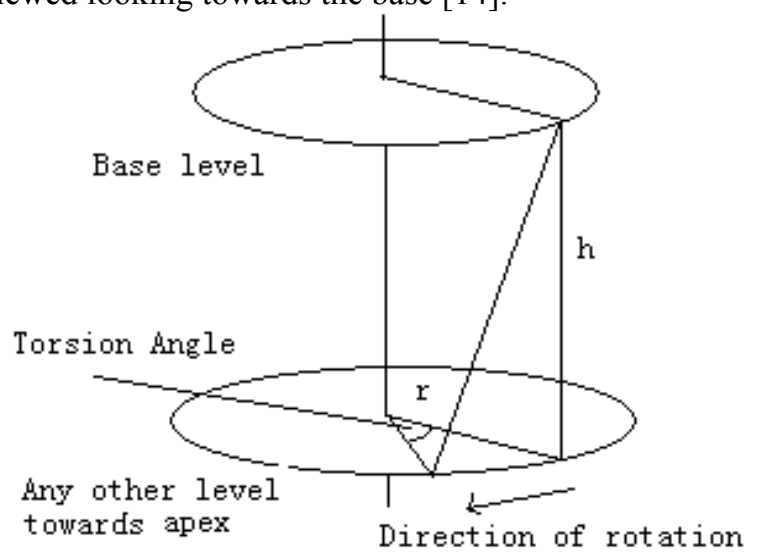

Fig. 3 Diagram illustrating the torsion angle

\section{Results}

\subsection{Displacement of RV}

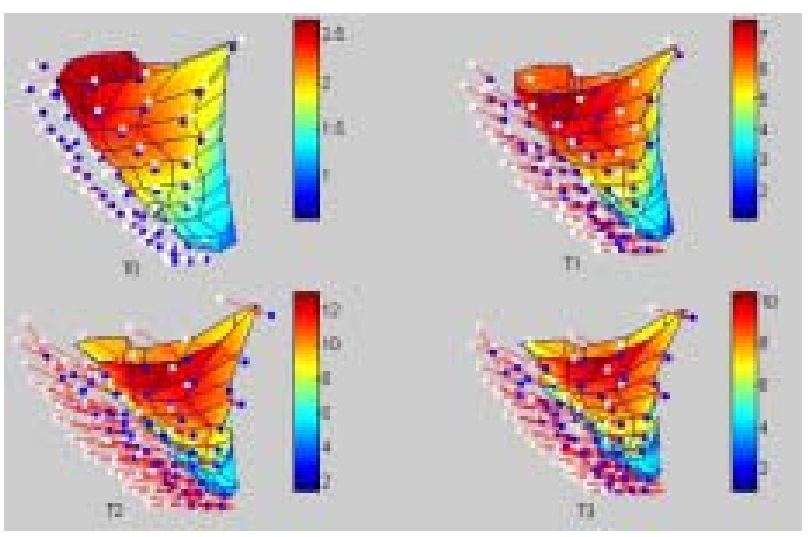

Fig. 4 The displacements of endocardium of RV at four different times (T0: end-isovolumetric contraction T1: middle-maximum ejection T2: end-maximum ejection T3: end-ejection)

Fig. 4 shows the displacements of endocardium of RV at four different times, the color bar denotes the length of 
displacements $(\mathrm{mm})$. The white dots mean the position of nodes at end-diastole, the red dots mean the position of nodes at different times through the systole, and the red line between the white dots and red dots mean the direction of nodes' displacements. We can see that there is a considerable displacement of the free wall towards the septum and a large displacement of the base towards the apex in the free wall, which contributed to a smaller RV cavity. The results are similar to those from the Tagged MRI [15].

\subsection{Minimum main strain of $\mathrm{RV}$}

Due to the complex shape of the RV, it is difficult to find a single axis about which to calculate twisting and radial contraction like those of LV. Here we use coordinate-system-independent principal strains and their directions to describe the deformation of RV. These quantities are derived by finding the eigenvalues and eigenvectors of the Lagrangian strain tensor, respectively. The eigenvalues of $\mathrm{E}$ are the principal strains, where a positive value indicates extension while a negative value indicates compression. The minimum principal strain, E3, is the smallest eigenvalue of $\mathrm{E}$, and along with the minimum principal strain directions, supplies potentially useful information about the magnitude and direction of contraction in the heart [16].

Fig. 5 shows the distribution of E3 at the endocardium of RV. The short red lines mean the directions of E3 of the central point of finite elements at the endocardium of $\mathrm{RV}$. We can see that the minimum principal strain is not uniform at the endocardium. A maximum value of -0.35 is at the apex and the directions of E3 mostly towards the apex, which means that the maximum contraction occurs towards the apex and in the approximate direction of the muscle fibers. The results are similar to those from the Tagged MRI $[15,17]$.
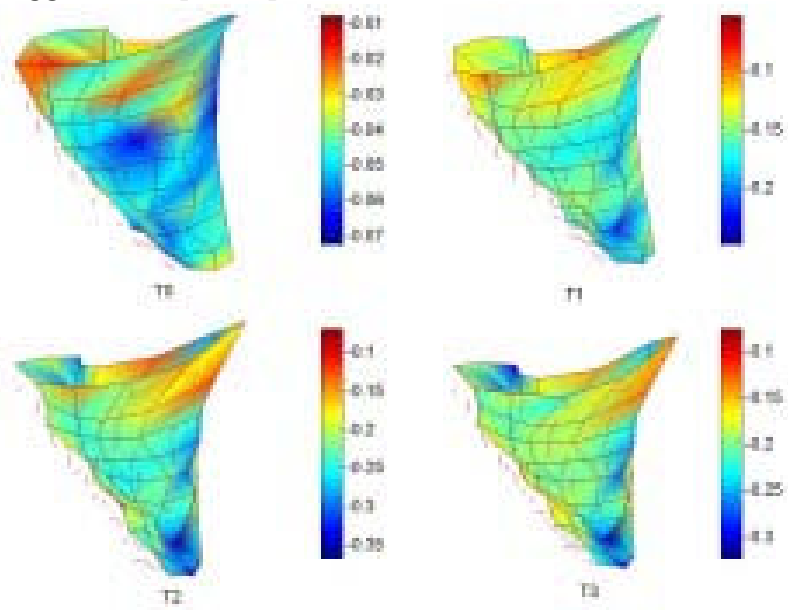

$\pi$

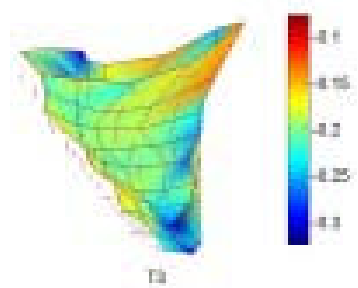

Fig. 5 The negative minimum principal strains and their directions of endocardium of RV at four different times (see Fig. 4)

\subsection{Displacement of $\mathbf{L V}$}

Fig. 6 shows the displacement and path of LV midwall along the long-axis. The color bar denotes the length of displacement $(\mathrm{mm})$. The white dots mean the position of nodes at different times through the systole, and the blue lines between the white dots mean the paths of nodes' displacements. We can see that the end-systolic longitudinal displacement magnitude increases from the apical to the equatorial levels and from the equatorial to the basal levels and the apex remains relatively fixed. We can also see that there is long-axis torsion which spiral from the apex to the base in a clockwise direction, as viewed from the base. The results are similar to those of literatures $[18,19]$.

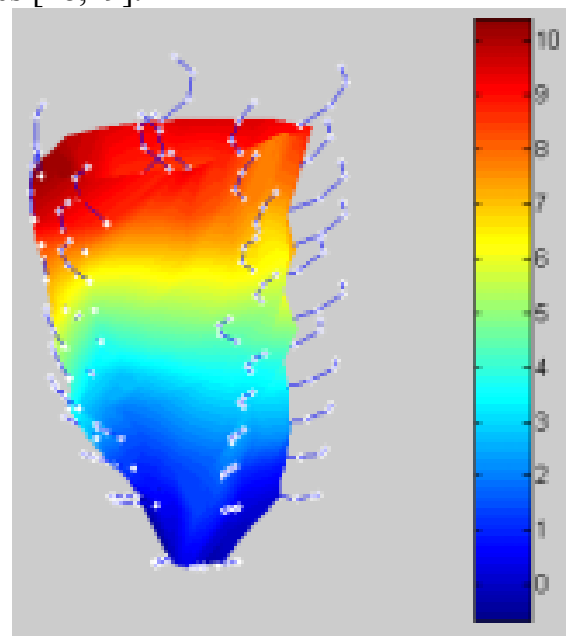

Fig. 6 the displacement of LV midwall

\subsection{Torsion angle of $\mathrm{LV}$}

Fig. 7 shows the variable curves of average torsion angle of LV midwall after certain spline interpolation. We can see that the torsion angle increases almost linearly towards the apex, which indicates that the counterclockwise rotation of the apex increases when viewed looking towards the base. In addition, along the radius direction, the torsion angles of endocardium are larger than those of midwall and epicardium, especially at the apex. The results are similar to those of literatures [20,21].

\section{Conclusions}

The ventricular wall motion and deformation caused by the cardiac electrical excitation was investigated based on a biventricular model. The results are in good accordance with solutions obtained from MR tagging images that reported in the literatures. This simulation suggests that such electromechanical biventricular model can be used to assess the mechanical function of two ventricles. The future work will investigate the interventricular dependence and ventricular wall relative motion based on the biventricular model. 


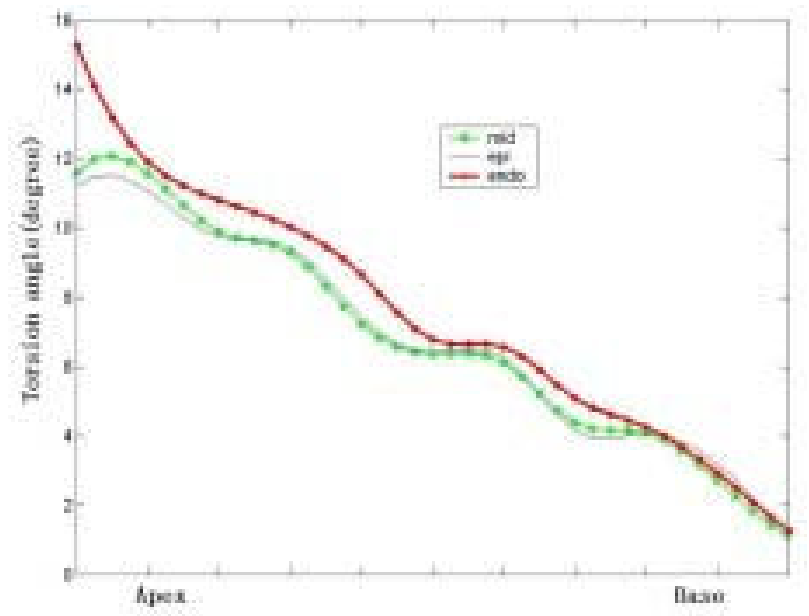

Fig. 7 The torsion angle of LV midwall

\section{Acknowledgements}

This project is supported by National Natural Science Foundation of China (30170243) and the Special Grant for the Authors of Excellent Doctoral Dissertation of Ministry of Education (199941).

\section{References}

[1] Sandler H, Alderman E. Left ventriclar tension and stress in man. Circ Res 1963; 13:91 104.

[2] Beyar R, Sideman S. A model of left ventricular contraction and transmural ventricular deformation. IEEE Computers in Cardiology 1990; 15 17.

[3] Nevo E, Lanir Y. Parameter estimation of left ventricular performance. IEEE Computers in Cardiology 1990; 251 254.

[4] Huyghe JM, Arts T, Van camoen DH, et al. Porous medium finite element model of the beating left ventricle. Am J Physiol 1992; 31: H1256 H1267.

[5] Guccione JM, Costa KD, McCulloch AD. Finite element stress analysis of left ventricular mechanics in the beating dog heart. J Biomechanics 1995; 28(10): 1167 1177.

[6] Bovendeerd PHM, Arts T, Huyghe JM, et al. Dependence of local left ventricular wall mechanics on myocardial fiber orientation: a model study. J Biomechanics 1992; 25: $1129 \sim 1140$

[7] Omens JH, May KD, McCulloch AD. Transmural distribution of three-dimensional strain in the isolated arrested canine left ventricle. Am J Physiol 1991; 261 (Heart Circ. Physiol. 30): H918-H928.

[8] Scollan DF, Holmes A, Winslow RL, et al. Histological validation of myocardial microstructure obtained from diffusion tensor magnetic resonance imaging. Am J Physiol
1998; 275: H2308-H2318.

[9] Nielsen PMF, Grice IJ, Smaill BH, et al. Mathematical model of geometry and fibrous structure of the heart. Am J Physiol 1991; 260: H1365 H1378.

[10] Lu W, Xia L. Computer simulation of epicardial potentials using a heart-torso model with realistic geometry. IEEE Trans Biomed Eng 1996;43:211-7.

[11] Liu F, Lu W, Xia L. The construction of three-dimensional composite finite element mechanical model of human left ventricle. JSME Int J 2001;44(C1):125-33.

[12] Spencer AJM, 1980. Continuum Mechanics. Longman, London.

[13] Buchalter MB, Weiss JL, Rogers WJ, et al. Non-invasive quantification of left ventricular rotational deformation in normal humans using magnetic resonance imaging myocardial tagging. Circulation 1990; 81;1236-1244.

[14] Guy AM, Daniel B, Walter JR, et al. Effects of afterload on regional left ventricular torsion. Cardiovascular Research 1996; 31: 917-925.

[15] Haber I, Metaxas DN, Axel L. Using Tagged MRI to Reconstruct A 3D Heartbeat. IEEE Computing In Science \& Engineering 2000,22-34.

[16] Haber I, Metaxas DN, Axel L Three-dimensional motion reconstruction and analysis of the right ventricle using tagged MRI, Medical Image Analysis 2000; 4: 335-355.

[17] Young, AA, Fayad ZA, Axel L. Right ventricular midwall surface motion and deformation using magnetic resonance tagging. Am.J PhysioL 1996; 271 (Heart Circ Physiol 40): $\mathrm{H} 2677-\mathrm{H} 2688$

[18] Moore CC, Lugo-Olivieri CH, McVeigh ER, et al. Threedimensional systolic strain patterns in the normal human left ventricle: characterization with Tagged MR Imaging. Radiology 2000; 214: 453-466.

[19] Rogers WJ, Shapiro EP, Weiss JL, et al. Quantification of and correction for left ventricular systolic long axis shortening by magnetic resonance tissue tagging and slice isolation, Circulation 1991; 84:721-731.

[20] Young AA, Imai H, Chang CN, Axel L. Two-dimensional left ventricular deformation during systole using magnetic resonance imaging with spatial modulation of magnetization. Circulation 1994; 89:740-752.

[21] Azhari H, Buchalter M, Sideman S, et al. A conical model to describe the nonuniformity of left ventricular twisting motion. Ann Biomed Eng 1992; 20:149-165.

Address for correspondence.

Ling Xia

Department of Biomedical Engineering

Zhejiang University, Hangzhou 310027, PR China

E-mail:xialing@hzcnc.com 